\title{
Regeneration of articular cartilage: Scaffold used in orthopedic surgery. A short handbook of available products for regenerative joints surgery
}

Alessandro Bistolfi ${ }^{*}$, Riccardo Ferracini ${ }^{2}$, Claudia Galletta ${ }^{3}$, Ferdinando Tosto ${ }^{3}$, Viola Sgarminato ${ }^{4}$, Elisa Digo ${ }^{4}$, Enrica Vernè ${ }^{4}$ and Alessandro Massè ${ }^{1,3}$

${ }^{1}$ AO Città della Salute e della Scienza, Department of Orthopeadics, Traumatology and Rehabilitative Medicine, CTO Hospital, Italy

${ }^{2}$ University of the Studies of Genova, IRCCS San Martino, Orthopaedics and Traumatology, Italy

${ }^{3}$ University of the Studies of Turin, Italy

${ }^{4}$ Polytechnic University of Turin, School of Engineering, Department of Science and Technology of Biomaterials, Italy

\begin{abstract}
Introduction: Restoring defects of load-bearing connective tissues such as articular cartilage resulting from traumas, degenerative or age-related diseases remains a significant clinical challenge for clinicians due to the limited inherent repair capacity of articular cartilage. Tissue engineering has emerged as a potential alternative to the traditional surgical techniques, as it can be effectively used to regenerate bone, cartilage and the bone-cartilage interface. Several scaffold strategies have been developed and evaluated for osteochondral defect repair.

Materials and methods: The classes of polymers (scaffold-based proteins, scaffold-base polysaccharides and synthetic scaffolds) and Hydrogels have been reviewed trough literature and market search. The study focused on their respective properties and analyzed advantages and disadvantages of each of them.

Discussion: Clinical studies demonstrated improved cartilage regeneration thanks to the implantation of biomaterials after bone marrow stimulation. New cartilage can be engineered in vivo by transplanting chondrocytes seeded into a three-dimensional scaffold and this novel scaffold has mechanical properties that can be comparable to native cartilage and could be used to repair large osteochondral joints defects. Anyway, there is still space for improvement regarding clinical outcome and tissue quality.
\end{abstract}

\section{Introduction}

Restoring defects of load-bearing connective tissues such as articular cartilage resulting from traumas, degenerative or age-related diseases remains a significant clinical challenge for clinicians due to the limited inherent repair capacity of articular cartilage. Articular cartilage defects are increasingly common among the adult population related to the Osteoarthritis $(\mathrm{OA})$ that throughout the years may lead to pain, reduced joint function and disability among affected patients [1]. Articular cartilage is a specialized tissue that covers joint surfaces and provides a low-friction and load-bearing surface for a smooth motion of joints. Healthy articular cartilage is able to withstand decades of repetitive loading that regularly exceeds several times body weight. Its unique behavior is attributed to the multiphasic nature of the extracellular matrix (ECM), which can be described as a charged, porous-permeable solid that is saturated with water and dissolved ions. Collagen, the primary component within the solid phase of the ECM, forms a fibrous network that is predominantly responsible for determining the tensile and shears mechanical characteristics of the tissue. Intermingled throughout this collagen microstructure are large, negatively charged proteoglycan macromolecules that give cartilage the ability to imbibe water and swell the tissue in an attempt to maintain physicochemical equilibrium. This swelling effect, which is constrained by the collagen network, pressurizes the tissue and contributes significantly to its load bearing and low-friction properties.
The structure and function of this tissue can be compromised by degeneration, advanced age, female sex, obesity, repeated joint microinjuries, or injection of chondrotoxic agents. Due to its avascular nature, damaged cartilage tissue does not heal spontaneously and it remains a challenge to fully restore tissue function. The poor capacity for self-repair of chondral defects has resulted in the development of a large variety of treatment approaches.

Bone marrow stimulation techniques such as microfracture and subchondral drilling involve the surgical placement of holes that connect the debrided cartilage lesion to the underlying bone marrow stroma to recruit pluripotential cells capable of repairing soft and hard tissues. Increased recruitment of marrow-derived stromal cells was linked to improved cartilage repair in rabbit models. Chondrogenic foci arising from subchondral bone progenitors have also been identified as the main source of cartilaginous repair tissue for the chondral

Correspondence to: Alessandro Bistolfi, AO Città della Salute e della Scienza, Department of Orthopeadics, Traumatology and Rehabilitative Medicine, CTO Hospital, Via Zuretti 29, 10126 Turin, Italy, Tel: +39-0116933573, Fax: +300116933760,E-mail: abistolfi@cittadellasalute.to.it

Key words: scaffold, osteoarthritis, cartilage, regenerative, tissue engineering

Received: October 31, 2017; Accepted: November 27, 2017; Published: November 30, 2017 
defects in animal models. Microfracture surgery may be applied to treat cartilage defects. During the procedure the subchondral bone is perforated, allowing bone marrow-derived mesenchymal stem cells to migrate towards the defect site and form new cartilage [2]. The latter strategy, also known as bone marrow stimulation including bone marrow stromal cells (BMSCs) and biodegradable scaffolds, is relatively simple, minimally invasive and inexpensive. Tissue engineering and regenerative medicine may improve the outcome of microfracture surgery. Filling the subchondral defect with a biomaterial may provide a template for the formation of new hyaline cartilage tissue $[3,4]$.

Tissue engineering has emerged as a potential alternative as it can be effectively used to regenerate bone, cartilage and the bonecartilage interface. Several scaffold strategies, such as single phase, layered, and recently graded structures have been developed and evaluated for osteochondral defect repair. Applying biomaterial scaffold whose primary function is to control the shape and volume of the newly formed tissue, it is possible to facilitate, at the same time, the attachment, proliferation, and differentiation of embedded cells [57]. In addition to influencing the shape and metabolic activity of the engineered construct, scaffolds must also be capable of withstanding the mechanical environment of the native tissue that has to be replaced.

\section{Materials and methods}

The matrices to repair cartilage defects can be realized with different types of materials:

- natural materials

- synthetic materials

- hydrogel

Natural materials have properties such as biodegradability and bioactivity, fundamental for the good integration with the host tissue. They also promote the growth and proliferation of cells.

The biomaterials can be based on proteins or polysaccharides.
The synthetic materials offer good control over the composition and consistency of the matrix, have mechanical properties and adjustable biodegradability and involve low cost of synthesis. Compared with natural materials, their degradation products may be harmful to the body. We distinguish the polyesters, which include poly (hydroxy acids) and poly (idrossialcanoati), and polyurethanes.

Hydrogels are constituted by viscous polymers obtained by covalent or ionic bonds between macromolecules hydrophilic. The hydrogels physically entrap the cells inside of the structure, which are distributed evenly. Their hydrophilicity reproduces the hydrated nature of the cartilage and allows to minimize the surgical invasiveness, as these materials are easily injectable. Listed in table 1 the classes of polymers reviewed in this manuscript.

\section{Scaffold-based proteins}

\section{MeRG - Biocollagen ${ }^{\circledR}$ (Biotech)}

Description: It is a resorbable membrane for guided tissue regeneration, used in the one-step techniques.

Composition: It consists of type I collagen lyophilized, hypoallergenic and extracted from equine Achilles tendon (Type I> $95 \%$, Type III $<5 \%$, glycosaminoglycans $2,75 \mathrm{mg} / \mathrm{g}$ ).

Preparation: It is advisable to rehydrate the product with sterile saline for 3-5 minutes

Sterilization: the product is disposable and non-sterilizable.

Property: It consists of a microfibrillar collagen membrane, nonfriable and resistant to traction and torsion.

The membrane has a smooth side and a rough-fibrillar side that allows fixing the matrix with fibrin glue. The interaction of collagen with thrombocytes is important for blood coagulation as the link with the platelet integrin determines the degranulation of the platelets themselves, and the "release" of factors activating the coagulation cascade with the formation of the fibrin lattice. The grid formed by

Table 1. Classes of Polymers and respective properties.

\begin{tabular}{|c|c|c|c|}
\hline Class of Polymers & Material & Advantages & Disadvantages \\
\hline \multirow[t]{2}{*}{ Based on Protein Polymers } & $\begin{array}{l}\text { Fibrin: From fibrinogen degradation, starring } \\
\text { role in coagulation }\end{array}$ & $\begin{array}{l}\text { Lower toxicity } \\
\text { Low cost } \\
\text { Promotion of adhesion and migration of cells }\end{array}$ & Poor biomechanical strength \\
\hline & $\begin{array}{l}\text { Collagen: Main component of the ECM, it } \\
\text { allows cells to maintain their phenotype. }\end{array}$ & $\begin{array}{l}\text { Help adhesion of cells } \\
\text { Biodegradable }\end{array}$ & $\begin{array}{l}\text { Physical and chemical variable properties } \\
\text { Variable degradation }\end{array}$ \\
\hline \multirow[t]{2}{*}{ Based on Polysaccharide Polymers } & $\begin{array}{l}\text { Hyaluronic acid: It maintains the degree of } \\
\text { hydration, firmness, plasticity and viscosity } \\
\text { of cartilage. }\end{array}$ & $\begin{array}{l}\text { Low toxicity } \\
\text { Supporting migration of mesenchymal stem } \\
\text { cells and epithelial cells } \\
\text { Fill irregular defects }\end{array}$ & Poor biomechanical strength \\
\hline & $\begin{array}{l}\text { Alginate: From algae, it absorbs water } \\
\text { quickly. }\end{array}$ & $\begin{array}{l}\text { Plentiful and available in nature } \\
\text { Biodegradable }\end{array}$ & $\begin{array}{l}\text { Slow degradation } \\
\text { Poor biomechanical strength } \\
\text { Not suitable for long-term implants }\end{array}$ \\
\hline \multirow[t]{4}{*}{ Synthetic Polymers } & $\begin{array}{l}\text { PLA: Chiral molecule used in many } \\
\text { commercial products. }\end{array}$ & $\begin{array}{l}\text { High stress resistance } \\
\text { High Young's modulus } \\
\text { Possibility of synthesizing in different forms }\end{array}$ & $\begin{array}{l}\text { His depolymerization require excessive } \\
\text { heating } \\
\text { Local acidosis caused by biodegradation } \\
\text { products }\end{array}$ \\
\hline & $\begin{array}{l}\text { PGA: Synthesized by condensation of } \\
\text { glycolic acid, used to prepare of absorbable } \\
\text { sutures }\end{array}$ & $\begin{array}{l}\text { Good mechanical resistence } \\
\text { High Young's modulus } \\
\text { His product of degradation (glycolic acid) } \\
\text { exists in nature }\end{array}$ & $\begin{array}{l}\text { Rapid degradation } \\
\text { His product of degradation (glycolic acid) } \\
\text { causes acidosis }\end{array}$ \\
\hline & $\begin{array}{l}\text { PCL: Amorphous structure, suitable to the } \\
\text { release of drugs }\end{array}$ & $\begin{array}{l}\text { Good osteoinductive potential } \\
\text { Non- toxic degradation products } \\
\text { Good mechanical properties }\end{array}$ & Acid residues as a result of degradation \\
\hline & $\begin{array}{l}\text { PLGA: amorphous and soluble in many } \\
\text { solvents }\end{array}$ & $\begin{array}{l}\text { Good mechanical resistance } \\
\text { Biodegradable and biocompatible } \\
\text { Resistant to hydrolysis }\end{array}$ & Amorphous, so it's difficult to use. \\
\hline
\end{tabular}


fibrin retains elements of the corpuscular part forming the clot that induce migration of fibroblasts.

Activity: MeRG favors the cell surface adhesion and holds mesenchymal cells, preventing the latter from escaping into the joint cavity.

Metabolization: MeRG derived from natural equine collagen, so it's reabsorbed spontaneously in 60/90 days.

Contraindications: known hypersensitivity to collagen.

\section{Chondro-Gide ${ }^{\bowtie}$ (Geistlich)}

Description: It's a double-layer collagen matrix; it's available in sizes $20 \times 30 \mathrm{~mm}, 30 \times 40 \mathrm{~mm}$ and $40 \times 50 \mathrm{~mm}$.

Composition: It is composed of type I and III collagen and it's presented as a collagen matrix bilayer. The compact layer prevents the spreading of mesenchymal stem in the joint and protects mechanical stress; the porous layer is made of dispersed collagen fibers that support the engraftment of the cells.

Preparation: It is necessary rehydrate the product with sterile physiological solution for 3-5 minutes.

Sterilization: The product is disposable and non resterilizable.

Property: Chondro-Gide is made by porcine collagen, which is naturally degraded into oligopeptides first and then into individual amino acids by collagenases, gelatinases and proteinases. Finally, it is easy to handle and prevents intra-articular bleeding.

Activity: The functional principle of Microfracturing is based on multipotent mesenchymal stem cells, cytokines and growth factors released by the subchondral bone.

The clot formed as a result of hemorrhage is stabilized by the matrix and the fibrin glue. At this point, Chondro-Gide constitutes a suitable support structure to promote chondrogenic differentiation of cells and, in combination with fibrin glue, stimulates chondrocytes to increase the deposit of proteoglycans.

Contraindications: The use of Chondro-Gide is contraindicated in patients with known hypersensitivity to collagen.

\section{Novocart 3D - AesculapOrthopaedics (BBraun)}

Description: It is a two-phase matrix used in 3D- ACT therapy (autologous chondrocyte transplantation tridimensional) to fill cartilage defects.

Composition: The material consists of a membrane coating (A) applied on the surface of a spongy structure with interconnected pores (A 'and A' '); the sponge and the membrane are solidly connected to one another. The matrix, based on type II collagen and aggrecan, is combined with physiological components of hyaline cartilage and subsequently autologous chondrocytes are added.

Preparation: The production is possible to use after three weeks after removal of the hyaline cartilage of the patient.

Property: Novocart-3D has high biomimetic and reduces the presence of inflammatory mediators (such as interleukin-1). Finally, as it uses an autogenous serum, avoids the use of antibiotics and antifungals.

Activity: The matrix keeps the cultured cells vital and synthesizes the proteins present in cartilage, which offer a decisive contribution to the tissue biomechanics. In addition, 3D Novocart induces the regeneration of cartilage through the growth and differentiation factors.

Indications: The cartilage defects treated with 3D Novocart derive from osteochondritis dissecting or trauma of the bearing regions of the joints. It is indicated in young patients (but not younger than 16 years) to avoid possible secondary damage such as premature osteoarthritis.

Contraindications: The use of $3 \mathrm{D}$ Novocart is contraindicated in patients with known hypersensitivity to collagen. It is also contraindicated in cases of cartilage defects caused by inflammatory diseases or massive degeneration. The defect must not be more extensive than $14 \mathrm{~cm} 2$ or deeper than $7 \mathrm{~mm}$.

\section{MaioRegenTM - Biojoint System}

Description: MaioRegen represents a new therapeutic concept inspired by nature that improves the regeneration of bone and cartilage. It is a three-dimensional matrix that mimics the osteo-cartilaginous tissue, composed of subchondral bone and cartilage. Easy to use and apply in one-step procedures, MaioRegen is available in $3,5 \times 3,5 \times 0,6$ and $5 \times 5 \times 0,6 \mathrm{~cm}$ formats.

Composition: It is presented as a multi-layer and monolithic scaffold: the surface layer is composed of equine collagen type I and reproduces the cartilage tissue, while the deepest layer consists of hydroxyapatite, rich in magnesium (Mg-HA), and simulates the structure subchondral. The intermediate layer, composed of Mg-HA and collagen, reproduces the interface between the two fabrics.

\section{Property:}

- Biomimetics: The particular chemical composition and structure makes MaioRegen a completely biomimetic scaffold. The "chemical" biomimetics is the result of the combination of collagen macromolecules and nanocrystals of $\mathrm{MgHA}$, which is done through an innovative nucleation mechanism. The "structural" biomimetics is due to the characteristic orientation of the collagen fibers, arranged as in the human osteochondral tissue.

- Hydration: MaioRegen can quickly absorb body fluids (water absorption: 75\%) for its high porosity and hydrophilicity. The thickness of the membrane provides a solid stabilization, without any suture or other fixation methods.

-Flexibility: MaioRegen is flexible and elastic and it is adapting with the anatomical curvature of the application site.

- Conformability: MaioRegen can be easily adapted during surgery to the size of the defect and can also be used for the treatment of extended lesions.

- Biodegradability: The chemical composition of biomimetic membrane allows its complete degradation, thus facilitating the regenerative processes.

Activity: The porous structure and the chemical composition of MaioRegen improve chemotaxis cell. Progenitor cells derived from bone marrow adhere to collagen fibers, colonizing the scaffold. At this point, the cells are able to proliferate, differentiate and synthesize the cartilaginous and bone matrix in accordance with the organic mineral gradient present in the scaffold.

Indications: MaioRegen is indicated in every case it is necessary to restore the connective tissue in the joints, in particular in the treatment of traumatic, post-traumatic or degenerative osteo-chondral lesions, or 
if caused by osteochondritis dissecans; in III-IV grade lesions according to the Outbridge's classification and in single or multiple focal lesions.

Contraindications: MaioRegen should not be used in patients with advanced arthritic conditions, immune disorders, infectious or neoplastic diseases, obesity and older than 60 years.

\section{Neocart $^{\oplus}$ (Histogenics)}

Description: It is a three-dimensional matrix that is disseminated with patient's cells (two-step technique). It's used to recreate a tissue similar to the hyaline cartilage of the knee.

Composition: It's composed of type I collagen extracted from bovine.

Preparation: The NeoCart system is realized using the chondrocytes of the patient, generally taken from the articular surface of the femur. The cells are expanded, grown into the scaffolds of collagen type I and then incubated in the TEP (Tissue Engeneering Processor). It's designated to simulate the variation of mechanical forces and to reduced oxygen pressure. The incubation in this environment allows the chondrocytes to maintain their phenotype and to produce the proteins of the extracellular matrix, essential for the cartilage functionality.

Property: The system (scaffold and chondrocytes) reproduces the architecture of the knee articular cartilage and performs its biochemical functions. NeoCart is biocompatible, bioresorbable and maintains over time its mechanical characteristics.

Contraindications: The membrane is not indicated in patients with known hypersensitivity to collagen.

\section{$\mathrm{CaReS}^{\varpi}$ (Arthrokinetics)}

Description: It is an innovative technology for the surgical treatment of joint injuries. It is a three-dimensional structure that reproduces hyaline cartilage through the autologous chondrocyte implantation. It is used in two-step procedures.

Composition: CaReS is a three-dimensional type I collagenmatrix cells (made for the $99.8 \%$ of pure native collagen), on which the autologous chondrocytes are cultivated.

Preparation: Once the biopsy is performed, chondrocytes are isolated and combined with fluid matrix. After 14 days, without growth factors, the material is fixed in the lesion by a fibrin glue to involve the cartilaginous structures adjacent to the articulation.

Property: The high functionality and cellular activity allow a natural production of type II collagen.

CaReS has a stable form and structure and its size can be easily adjusted. The height and thickness can be adapted to the lesion.

Activity: It accelerates and optimizes the regeneration process of hyaline cartilage, through the production of type II natural collagen. Chondrocytes grown on Cares, in fact, are able to proliferate thanks to the highly bioactive support and capable of releasing growth factors.

Indications: The use of CaReS is indicated for lesions sized between 2.5 and $10 \mathrm{~cm} 2$ and III and IV grade according to Outerbridge's classification. It is also recommended in case of osteochondritis dissecans.

Contraindications: CaReS is contraindicated in case of damaged ligaments, rigid joints, arthrofibrosis, inflammatory processes, infectious diseases, metabolic disorders and in patients older than 60 years.

\section{CartiMaix $^{\circledR}$ (Matricel)}

Description: CartiMaix is a biodegradable membrane used as a carrier in the cartilage regeneration techniques in both one-step and two-step techniques.

It's available in three sizes: 25x30 mm, 30x40 mm and 40x50 mm.

Composition: The scaffold is made of porcine collagen and elastin and has two layers: the first one is thick and it interfaces with the joint space providing a protective shield; the second one is fibrous and it's in contact with the cartilage defect, providing an ideal matrix for the cells adhesion.

Preparation: The membrane is used in one-step procedure MAMFX (MatrixAssisted Bone MarrowStimulation) for lesions with dimensions comprised between 2 and $12 \mathrm{~cm} 2$ : the imprint of the lesion is taken after a diagnostic arthroscopy, using the aluminum template provided and then the membrane is properly shaped. The membrane is fixed with the fibrous side in contact with the damaged site, after subchondral bone perforations, through the fibrin glue. The correct positioning and its stability are finally verified by subjecting the articulation in flexion and extension movements.

The selected technique is called CACI (Collagen-covered Autologus chondrocyte Implantation) in case of $12 \mathrm{~cm} 2$ wider lesions. It involves two steps: the patient's autologous chondrocytes are picked and expanded in the laboratory under controlled conditions to form a suspension, then are injected into the defect. The correct positioniting of the matrix is checked at the end of the procedure testing the knee movement.

Sterilization: The membrane is provided inside a double-sterilized blister, accompanied by an aluminum sterile template $(40 \times 50 \mathrm{~mm})$ that can be employed to create the imprint of the defect and using it to obtain the proper shape.

Property: Scaffold is based on collagen and elastin so it's safe and biocompatible.

Contraindications: Cartimaix is controindicate in case of known hypersensitivity to one of the product components.

\section{Scaffold-based polysaccharides}

\section{Hyalofast ${ }^{\circledR}$ (Anika)}

Description: It is a biodegradable scaffolds used in the repair of chondral defects, through a one step procedure. It is available in $2 \times 2$ $\mathrm{cm}$ and $5 \times 5 \mathrm{~cm}$ sizes.

Composition: It is constituted by a single three-dimensional fibrous layer of $\mathrm{HYAFF}^{\oplus}$, a benzyl ester of hyaluronic acid.

Preparation: HyaloFast system can be implanted with miniartrotomies. The scaffold is cut with sterile scissors and placed on the lesion with the help of pliers after assessing the size of the defect through a template. If necessary, it can be fixed by fibrin glue or sutures. Several layers can be used in case of very deep lesions.

Sterilization: The sterilization is obtained by means of gamma rays.

Property: HyaloFast can be adapted to the size of the lesion and it's easily applied arthroscopically because it presents a bioadhesive nature. The degradation time is controllable and it's bioresorbable because it has the natural configuration of the hyaluronic acid. Thanks to the absence of animal or human derivated, the risk of inflammatory response is reduced. 
Activity: HyaloFast provides a biodegradable support for the engraftment and immobilisation in situ of mesenchymal stem cells, originating from the bone marrow after microfractures.

Warnings: The membrane is exclusively for surgical use, it should be stored in a cool, dry place $\left(\mathrm{T}<40^{\circ} \mathrm{C}\right)$ and requires immediate use after package opening.

\section{Hyalograft $\mathrm{C}^{\circledast}$ (Anika)}

Description: It is an advanced therapeutic product which it containing patient's cartilage cells included in $2 \times 2 \mathrm{~cm}$ inserts. Each insert contains approximately $4 \times 10^{\wedge} 6$ autologous and vital chondrocytes. It is used in two-step techniques for the damage repair in femoral condyles and trochlea cartilage.

Composition: Autologous chondrocytes are expanded in vitro and cultured on the scaffold. The scaffold is composed by a threedimensional structure to the fibrous layer $\mathrm{HYAFF}^{\bullet}$, which is a esterified derivative of hyaluronic acid.

Preparation: A arthroscopic biopsy of healthy cartilage from patient's knee is taken. The chondrocytes are isolated and expanded in vitro before being seeded in the scaffold of hyaluronic acid. Hyalograft $\mathrm{C}$ is then implanted by arthroscopy or arthrotomy.

Property: It is easily adaptable to the lesion size thanks to its solid consistency and intrinsic adhesive properties. The scaffold fibers have a $20 \mu \mathrm{m}$ diameter and a variable pore size.

Activity: The product is suitable for surgical repair of cartilage defects caused by sudden or repeated micro-traumas in adults and young people (once the cartilage growth is completed).

Contraindications: The use of Hyalograft $\mathrm{C}$ is contraindicated in patients with known hypersensitivity to the product components.

Warnings: In January 2013 Hyalograft $C$ was withdrawn from the market because of the outcome of the preliminary assessment of the CAT (Committee of Advanced Therapies).

\section{Cartipatch $^{\oplus}$ (Xizia Biotech)}

Description: Cartipatch is composed by a single layer of expanded cartilage cells combined with a hydrogel. The aim is improving the stability of the cells phenotype and simplify the surgical positioning.

The scaffold has a cylindrical shape, with a smooth end to reproduce the cartilage surface and a cone base to facilitate the installation. The available diameters are $10 \mathrm{~mm}, 14 \mathrm{~mm}$ or $18 \mathrm{~mm}$ and a thickness is 4 $\mathrm{mm}$. Used in two-step techniques.

Composition: The scaffold is made combining agarose and alginate; alginate provides an elasticity to the matrix which improves the handling during the surgery.

Preparation: $200 \mathrm{mg}$ of cartilage is collected arthroscopically. Cells are extracted through enzymatic digestion and they are grown in a bioreactor with the addition of autologous serum and are multiplied (up to about 10 million per $\mathrm{ml}$ ). Cells are then isolated and placed inside an agarose and alginate gel; the suspension is molded to obtain the cylindrical implants of different diameters. The hydrogel grows for about 21 days with a growth factor and undergoes a phenotype and vitality test. At this point the graft is surgically implanted: A proper cavity should be created with a drill to accommodate the scaffold and then the matrix is positioned by a needle. The needle has a dual function: to spill the captive air in the cavity and center the defect site.

\section{Sterilization: Obtained by autoclave.}

Property: The development of Cartipatch followed the aim to improve the ease and speed of the surgical gesture, guarantee a homogeneous distribution of the cells within the lesion and absence of risk of grafted chondrocyte cell outdistance. The inclusion of the cells within a three-dimensional matrix can meet all these demands. Cartipatch is biocompatible (guaranteed by the absence of animal products) and permits the cell differentiation. This requirement is obtained thanks to an appropriate three-dimensional environment and also using an autologous serum instead of a bovine serum as a growth factor. The hydrogel, combined with a cell suspension, allows to model complex shapes at $37^{\circ} \mathrm{C}$.

\section{Cartipatch ensures a longtime vitality of the cells.}

Indications: The Cartipatch system requires three-dimensional instruments for the different measures of matrix $(10,14$ and $18 \mathrm{~mm})$.

Contraindications: Do not use Cartipatch in case of known hypersensitivity to one of the product components.

\section{Synthetic scaffolds}

\section{Chondrotissue $^{\circledast}$ (Biotissue)}

Description: It is a medical device class III CE, used in treatments without autologous cells (one-step technique), for cartilage defects in the knee, hip or ankle. It is white-coloured, consistency is firm and different sizes are available (20x30x1,1 mm).

Composition: Chondrotissue is a membrane based on PGA and hyaluronic acid.

Preparation: The hyaluronic acid is freeze-dried with the polyglycolic acid (PGA) base, then packed.

Sterilization: Chondrotissue must be created in sterile conditions. Hyaluronic acid is used, sterilized by moist heat and felt in PGA, sterilized by radiation. It is a disposable product because its properties can be altered in a re-sterilization.

Property: The matrix is biocompatible and resorbable because of the PGA felt, that dissolves without residue in a few weeks in order to make way for the new tissue. PGA does not contain animal or human components and it is ideal for patients with allergies to animal protein.

Chondrotissue resurfaces and fill in the lesion and promotes the cartilage repair, relieves pain, and improves mobility. This scaffold offers maximum stability and great joint strength. Thanks to its texture, it can permit a hemostasis function in case of local bleeding, which can be occured in case of microfractures.

Indications: In order to restore the elastic properties, it is recommended to mix chondrotissue with autologous plasma or serum enriched with platelets (PRP) before implantation. If these are not available, the elasticity can be restored by infusion of physiological saline solution.

The scaffold can be molded to the size of the defect and it may be fixed by resorbable "pins" or suture thread. It can also be pasted on the defect by fibrin glue, which should be applied on the membrane edge.

Chondrotissue should be stored in a cool and dry place, protected from sunlight and keep away from sources of radiation.

Contraindications: Chondrotissue is contraindicated in case of known hypersensitivity to one of the ingredients of the product and 
should not be used in patients who must undergo a chemotherapy and/ or radiotherapy within three weeks from implantation.

\section{Bioseed $^{\oplus}$ - C (Biotissue)}

Description: BioSeed-C is constituted by a rectangular matrix whose length, width and thickness respectively correspond to $30 \times 20$ x $2 \mathrm{~mm}, 30 \times 30 \times 1.1 \mathrm{~mm}, 30 \times 20 \times 1.1 \mathrm{~mm}$ or $50 \times 20 \times 1.1 \mathrm{~mm}$. It contains intercommunicating cavities where 28.8 million autologous cells are uniformly distributed. The cells were previously isolated from a patient's cartilage sample, multiplied in vitro for 3 days of culture, and finally fixed to the membrane fibrin (two-step technique).

Composition: The matrix is made from a resorbable polymeric material of pure polyglycolic acid or from a composite of polyglactin 910 and poly-p-dioxanone (PDS). The material is free of dyes, soft and elastic, and it can be flexed and cut without fraying.

Preparation: Polyglactin 910 and PDS are combined together using a thermoplastic process without use of additives or excipients. The obtained matrix has pores able of retaining the cells and promoting the growth.

Sterilization: BioSeed-C can be stored, after inoculation of autologous chondrocytes, in its sterile packaging up to the time of transplantation and kept inside the insulating container. The 96 hours of validity of the product are guaranteed at a temperature between $4^{\circ}$ and $25^{\circ} \mathrm{C}$.

Property: BioSeed-C replaces the cartilage defects and restore its functions thanks to th perfect three-dimensional chondrocytes scaffold. The stability of the environment stimulates these cells to differentiate. The major advantage is the use of the patient's cells: BioSeed C cannot reject and it is therefore biocompatible and bioresorbable.

Indications: Underlying diseases must be checked before the blood sample. Affected cartilage must be completely removed before implantation, avoiding to damage the underlying bone plate.

BioSeed-C consistency allows the transport and the application with tweezers or by hands, but requires occasionally immersion in physiological saline solution to prevent drying. The membrane must be shaped according to the lesion size. It can be inserted with one of the traditional surgical techniques and fixed with sutures, absorbable tacks or fibrin glue.

Contraindications: BioSeed-C is contraindicated in case of hypersensitivity to bovine proteins or to substances present in the product. It could have minimal traces of penicillin, amphotericin $B$ and residues of gentamicin. BioSeed-C must not take contacts with oxidant substances such as iodine and $\mathrm{H} 2 \mathrm{O} 2$ because they could denature proteins with heavy metals and they can be detrimental to cell viability and undermine the effectiveness of the product.

\section{Hydrogel}

\section{BioCartTMII (ProChon Biotech)}

Description: BioCart II is used in the two-step techniques for repairing the articular cartilage: the combination of autologous cells, embedded within a natural hydrogel, is a safe therapeutic approach.

Composition: The scaffold is made of fibrin and hyaluronic acid of human origin and it has a porous structure that allows the distribution of the cells.

Preparation: The hydrogel is prepared by combining a solution of human fibrinogen hyaluronic acid and thrombin in order to obtain the coagulum. It's lyophilized (freeze-drying) to create a porous scaffold with open channels for seeding the cells. The autologous chondrocytes from healthy cartilaginous tissue are expanded by enzymatic degradation of collagenase and they are expanded in culture with autologous serum and FGF in addition. The modified chondrocytes are added in the scaffold and implanted into the defect site.

Property: The scaffold is made by fibrin and hyaluronic acid, which make the product biocompatible and biodegradable. Then porous structure and the channels permit the distribution of cells homogeneously. BioCart II can promote the repair of the entire cartilage layer. Moreover, the chondrocytes are endowed with a higher proliferation rate than non-modified cells thanks to the growth factor used.

Contraindications: BioCart II is controindicated in case of known hypersensitivity to one of the components of the product.

\section{Discussion}

The regeneration of damaged cartilage using implantation of acellular biomaterials has been widely investigated using preclinical models. Different outcome measures such as macroscopic and histological evaluation, semi-quantitative macroscopical and histological evaluation using scoring systems, histomorphometry, PCR and biochemical assays were used to assess the regenerative potential of implanting biomaterials. The general findings of the majority of the systematic review and meta-analysis are that the implantation of biomaterials improves cartilage regeneration compared to non-treated osteochondral defects [8]. In general, clinical studies demonstrated improved cartilage regeneration thanks to the implantation of biomaterials after bone marrow stimulation, but there is still space for improvement regarding clinical outcome and tissue quality. The autologous chondrocyte implantation (ACI) is a surgical approach used to treat full-thickness cartilage defects mainly in knee joints. Small samples of normal cartilage with chondrocytes is collected from the damaged joint. The cells are cultured in special laboratories to increase their number and reimplanted a few weeks later into the areas of cartilage damage. The aim of this procedure is to restore normal hyaline cartilage to the bones extremities and thereby restore normal joint function. The hope is that the stem cells from the marrow will differentiate into chondrocytes that will then produce new cartilage [9]. Filardo et al. described the implantation of an osteochondral biomimetic scaffold consisting of a type I collagen cartilage-like layer, a type I collagen/hydroxyapatite intermediate layer, and a mineralized blend of type I collagen and hydroxyapatite as a subchondral bone compartment, to treat patients with osteochondritis dissecans. Clinical scores improved significantly in these patients after the first two years and evaluation by MRI indicated good defect filling and implant integration, but also heterogeneous tissue regeneration and changes in the subchondral bone [10]. Chen et al directly compare the early repair response of cartilage defects in different anatomic locations using a bilateral model with defects created in both trochlea (TR) and medial femoral condyle (MFC). They found that bone marrow stimulation induced greater chondrogenesis in TR vs MFC defects in adult rabbits [11]. Furthermore Constance et al. underlined how cartilage tissue engineering is an interesting method with consistent regenerative potential to form a durable hyaline repair cartilage [12]. A variety of scaffolds, in combination with chondrogenic cells, have been shown to have good ability to regenerate hyaline cartilage both experimentally and clinically.

Therefore several studies have shown that new cartilage can be engineered in vivo by transplanting chondrocytes seeded into a three- 
dimensional scaffold. These studies demonstrated that a novel scaffold has mechanical properties that can be comparable to native cartilage and could be used to repair large osteochondral joints defects. However longer outcomes term are required in order to best select the proper treatment for osteoarthritis.

\section{References}

1. Jackson DW, Simon TM, Aberman HM (2001) Symptomatic articular cartilage degeneration: the impact in the new millennium. Clin Orthop Relat Res: S14-25. [Crossref]

2. Breinan HA, Martin SD, Hsu HP, Spector M (2000) Healing of canine articular cartilage defects treated with microfracture, a type-II collagen matrix, or cultured autologous chondrocytes. J Orthop Res 18: 781-789. [Crossref]

3. He A, Liu L, Luo X, Liu Y, Liu Y, et al. (2017) Repair of osteochondral defects with in vitro engineered cartilage based on autologous bone marrow stromal cells in a swine model. Sci Rep 7: 40489.

4. Brix M, Kaipel M, Kellner R, Schreiner M, Apprich S, et al. (2016) Successful osteoconduction but limited cartilage tissue quality following osteochondral repair by a cellfree multilayered nano-composite scaffold at the knee. Int Orthop 40: 625-632. [Crossref]

5. Ahn JH, Lee TH, Oh JS, Kim SY, Kim HJ, et al. (2009) Novel hyaluronate-atelocollagen beta-TCP-hydroxyapatite biphasic scaffold for the repair of osteochondral defects in rabbits. Tissue Engineering Part A 15: 2595-2604. [Crossref]
6. Athanasiou K, Korvick D, Schenck Jr (1997) Biodegradable implants for the treatment of osteochondral defects in a goat model. Tissue Engineering 3: 363-373.

7. Aulin C, Jensen-Waern M, Ekman S, Hägglund M, Engstrand T, et al. (2013) Cartilage repair of experimentally 11 induced osteochondral defects in New Zealand white rabbits. Lab Anim 47: 58-65. [Crossref]

8. Bal BS, Rahaman MN, Jayabalan P, Kuroki K, Cockrell MK, et al. (2010) In vivo outcomes of tissue-engineered osteochondral grafts. J Biomed Mater Res B Appl Biomater 93: 164-174. [Crossref]

9. Cui YM, Wu J, Hu YY (2009) Repairing articular cartilage defects in rabbits using bone marrow stromal cell-derived chondrocytes compounded with poly(lactic-co-glycolic acid). Journal of Clinical Rehabilitative Tissue Engineering Research 13: 1004910054 .

10. Filardo G, Kon E, Di Martino A, Busacca M, Altadonna G, et al. (2013) Treatment of knee osteochondritis dissecans with a cell-free biomimetic osteochondral scaffold: clinical and imaging evaluation at 2-year follow-up. Am J Sports Med 41: 1786-1793. [Crossref]

11. Chen H, Chevrier A, Hoemann CD, Sun J, Lascau-Coman V, et al. (2013) Bone marrow stimulation induces greater chondrogenesis in trochlear vs condylar cartilage defects in skeletally mature rabbits. Osteoarthritis Cartilage 21: 999-1007. [Crossref]

12. Chu CR, Szczodry M, Bruno S (2010) Animal Models for Cartilage Regeneration and Repair Tissue Eng Part B Rev 16: 105-115. [Crossref]

Copyright: $(02017$ Bistolfi A. This is an open-access article distributed under the terms of the Creative Commons Attribution License, which permits unrestricted use, distribution, and reproduction in any medium, provided the original author and source are credited. 